\title{
THE QUANTUM BINARY AND TRIPLET DISTRIBUTION FUNCTIONS FOR TWO-COMPONENT PLASMA
}

\section{A. EISA}

Department of Mathematics

Faculty of Education

Assuit University

New Valley

Egypt

e-mail: dalia_ah@yahoo.com

\begin{abstract}
The aim of this paper is to calculate the analytical form of the quantum binary and triplet distribution functions of charged particles that interact through the effective potential for two component plasma. We consider only the thermal equilibrium plasma in the case of $n \lambda_{a b}^{3}<<1$, where $\lambda_{a b}=\sqrt{\frac{\hbar^{2}}{m_{a b} K T}}$ is the thermal De Broglie wave-length; our calculations are based on the BogoliubovBorn-Green-Kirkwood-Yvon (BBGKY) hierarchy. Two forms of the quantum triplet distribution function are calculated; one of them is based on the Kirkwood superposition approximation (KSA) which is consisting of the assumption that the potential in a set of three particles is the sum of the three pair potentials, and the other form is calculated by integration the triplet distribution function.
\end{abstract}

2010 Mathematics Subject Classification: 82B05, 82B10, 82B30.

Keywords and phrases: the quantum binary and triplet distribution functions, the effective potential, the Slater sum.

Received November 20, 2015

(C) 2016 Scientific Advances Publishers 


\section{Introduction}

The calculation of the equilibrium properties of many-particle systems for a given type of microscopic interaction is one of the most fundamental problems in statistical physics. Strongly coupled plasmas are of great interest for the physics of matter under extreme conditions. Astrophysical problems such as the description of stellar and planetary interiors have stimulated this interdisciplinary field from the beginning. Strongly coupled plasmas are intensively studied in material sciences, physical chemistry, condensed matter and high-pressure physics, and as a special topic in plasma physics. In addition, fundamental problems of many-particle physics are closely related to this field. Charged particles as the elementary constituents of plasmas, e.g., electrons and ions or electrons and holes in semiconductors, interact via the long-range Coulomb potential so that many-particle effects such as dynamical screening and self-energy, Pauli blocking and degeneracy, dynamical local-field effects and structure factor have to be treated. Therefore, the precise diagnostics of strongly coupled plasmas gives us a tool to test several concepts of theoretical physics and in particular quantum statistics. Today the physics of strongly coupled plasmas is of outstanding relevance for major projects in inertial confinement fusion research using high-power lasers or intense heavy ion beams. Plasmas in unusual situations as in traps or in dimensionally reduced semiconductor structures gain in importance. Many fundamental but also demanding technical problems are closely related to the physics of dense plasmas. The high temperature plasmas are considered to dealt with the classical statistical mechanics, if their density is so low and their temperature is so high that they may be considered as fully ionized. However, the presence of the infinite attraction between positive and negative charges introduces difficulties when investigated by the classical statistical mechanics because the classical Boltzmann factor is infinite for the state where positive charge coalesces to a negative charge, leading to the divergence of the partition function. This divergence can be avoided by 
means of the quantum mechanical treatment. The quantum-statistical treatment of a system of charged particles has been given by several authors. The quantum correction identical to the classical distribution functions can be expressed by Slater sum with regard to the electronelectron and electron-ion interaction. Modern statistical mechanics have put the theoretical analysis of correlations on a firm ground. The binary and triplet distribution functions are one of the most important functions of statistical mechanics. The importance of the radial distribution function in statistical mechanics is due to the fact that all the thermodynamic quantities such as the pressure, the internal energy, and the free energies, etc. can be calculated from it. There are several closure approximations, which express the triplet distribution function in terms of the pair distribution function; such as Kirkwood superposition approximation (KSA) [1]. Many authors have studied the binary and triplet distribution functions [2-8]. The model under consideration is the two component plasma (TCP), i.e., neutral system of point like particles of positive and negative charges. For the numerical calculation, we restrict ourselves to the case of two component plasma which antisymmetric with respect to the charges $e_{e}=-e_{i}=-e$ and therefore symmetrical with respect to the densities $n_{i}=n_{e}=n$ further, the theoretical investigations are carried out for arbitrary electron ion mass ratios. To simplify the numeric investigations, we simulated so far only mass symmetrical (no relativistic) electron-positron plasma with $m_{e}=m_{i}=m$. We study this so far unrealistic case of mass-symmetrical plasmas in order to save computer time in particle simulations. The mass-symmetrical model is well suited to check the quality of various analytical approximations.

This paper is organized as follows. In Section 2, we obtain the binary distribution function. The quantum binary distribution function for two component plasma is given in Section 3. The quantum triplet distribution function is given in Section 4. The quantum triplet distribution function for two component plasma is given in Section 5. 


\section{The Quantum Binary Distribution Function (BDF)}

The quantum binary distribution function in the cluster expansion is given by [9] which we can rewrite it by using Mayer function in the following form:

$$
\begin{aligned}
F_{a b}^{q u}\left(r_{a b}\right)= & S_{a b} \exp \left(g_{a b}+\beta V_{a b}\right)\left[1+\sum_{c} n_{c} \int\left[f_{a c} f_{b c}-g_{a c} g_{b c}\right] d r_{c}\right. \\
& +\frac{1}{2} \sum_{c} \sum_{d} n_{c} n_{d} \iint\left[f_{a c} f_{b c} f_{a d} f_{b d} f_{c d}+f_{a c} f_{b c} f_{a d} f_{b d}+4 f_{a c} f_{b c} f_{c d} f_{b d}\right. \\
& +2 f_{a c} f_{c d} f_{b d}+2 g_{a c} g_{b d} g_{c d}+g_{a c} g_{b c} g_{a d} g_{b d}-4 f_{a c} g_{b d} g_{c d} \\
& \left.\left.-4 f_{b d} g_{a c} g_{c d}\right] d r_{d} d r_{1}+\ldots\right],
\end{aligned}
$$

where $f_{a b}=S_{a b} \exp \left(g_{a b}+\beta V_{a b}\right)-1$ is the Mayer function, $g_{a b}=\frac{l_{a b}}{r}$ $\exp (-\chi r)$ is the Debye potential, $V_{a b}=\frac{l_{a b}}{\beta r}, l_{a b}=\frac{e_{a} e_{b}}{D K T}, T$ is the absolute temperature, $K$ is the Boltzmann's constant, $e_{a}$ is the charge of particle $a, D$ is the dielectric constant, $\chi^{2} \frac{4 \pi}{D K T} \sum_{a} n_{a} e_{a}^{2}$ is the square of the Debye length.

We know that

$$
f_{a b}=\frac{1}{\sqrt[3]{2 \pi}} \int \exp (i k \cdot r) \widetilde{F}(t) d t
$$

then we have

$$
\begin{aligned}
& \int d r_{c} f_{a c} f_{b c} \\
& \quad=\frac{1}{(2 \pi)^{3}} \iiint d t_{1} d t_{2} d r_{c}\left[\widetilde{F}\left(t_{1}\right) \widetilde{F}\left(t_{2}\right) \exp \left(i t_{1} \cdot r_{a c}+i t_{2} \cdot r_{b c}\right)\right] \\
& \quad=\frac{1}{(2 \pi)^{3}} \iiint d t_{1} d t_{2} d r_{c} \exp \left(i\left(t_{1}+t_{2}\right) \cdot r_{c}\right) \tilde{F}\left(t_{1}\right) \tilde{F}\left(t_{2}\right) \exp \left(-i\left(t_{1} \cdot r_{a}+t_{2} \cdot r_{b}\right)\right)
\end{aligned}
$$




$$
\begin{aligned}
& =\frac{1}{(2 \pi)^{3}} \int d t_{1} d t_{2} \delta\left(t_{1}+t_{2}\right) \tilde{F}\left(t_{1}\right) \tilde{F}\left(t_{2}\right) \exp \left(-i\left(t_{1} \cdot r_{a}+t_{2} \cdot r_{b}\right)\right) \\
& =\frac{1}{(2 \pi)^{3}} \int d t_{1} \tilde{F}\left(t_{1}\right) \tilde{F}\left(-t_{1}\right) e^{i t_{1} \cdot r_{a b}}=\frac{1}{2 \pi^{2}} \int_{0}^{\infty} d t_{1} \frac{t_{1} \sin \left(t_{1} r_{a b}\right)}{r_{a b}} \tilde{F}\left(t_{1}\right) \tilde{F}\left(-t_{1}\right) .
\end{aligned}
$$

The quantum binary distribution function up to $O\left(e^{6}\right)$ in the following form:

$$
\begin{aligned}
F_{a b}^{q u}\left(r_{a b}\right)= & S_{a b} \exp \left(g_{a b}+\beta V_{a b}\right) \\
& \times\left[1+\frac{1}{(2 \pi)^{3}} \sum_{c} n c \int \frac{t \sin \left(t r_{a b}\right)}{r_{a b}}\{\widetilde{F}(t) \widetilde{F}(-t)-\widetilde{g}(t) \widetilde{g}(-t)\} d t\right. \\
& +\frac{1}{2} \sum_{c} \sum_{d} n_{c} n_{d}\left[l_{a c} l_{b d} l_{c d} \int \frac{t \sin \left(t r_{a b}\right)}{r_{a b}} \widetilde{g}^{2}(t) \widetilde{g}(-t) d t-l_{a c} l_{b c} l_{a d} l_{b d}\right. \\
& \left.\left.\times\left(\int d t \frac{t \sin \left(t r_{a b}\right)}{r_{a b}} \widetilde{g}(t) \widetilde{g}(-t)\right)^{2}\right]+\ldots\right]
\end{aligned}
$$

where

$$
\begin{gathered}
\tilde{g}_{a b}(t)+\beta \tilde{V}_{a b}(t)=\frac{4 \pi \chi^{2} l_{a b}}{t^{2}\left(t^{2}+\chi^{2}\right)} \\
\widetilde{S}_{a b}(t)=(2 \pi)^{3} \delta(t)-4 \pi \beta e_{a} e_{b} \frac{1}{t^{2}} e^{-\frac{1}{4} \lambda_{a b^{2}}^{2} t^{2}}{ }_{1} F_{1}\left(\frac{1}{2}, \frac{3}{2} ; \frac{1}{4} \lambda_{b}^{2} t^{2}\right) \\
+\delta_{a b} \frac{(-1)^{2 s}}{2 s+1} \sqrt{\pi^{3}} \lambda_{a b}^{3} e^{-\frac{1}{4} \lambda_{a b}^{2} t^{2}} \\
\times\left\{1-\frac{\beta e_{a} e_{b}}{\sqrt{\pi} \lambda_{a b}}{ }_{2} F_{2}\left(\frac{1}{2}, \frac{1}{2}, \frac{3}{2}, \frac{3}{2} ; \frac{1}{4} \lambda_{b}^{2} t^{2}\right)\right\}+\ldots
\end{gathered}
$$


After performing the integration, we get the quantum binary distribution function in the form

$$
\begin{aligned}
F_{a b}^{q u}\left(r_{a b}\right)= & {\left[1-\frac{1}{2} \delta_{a b} \exp \left(-\frac{r_{a b}^{2}}{\lambda_{a b}^{2}}\right)+\frac{l_{a b}}{r}\left\{1-\exp \left(-\frac{r_{a b}^{2}}{\lambda_{a b}^{2}}\right)\right\}\right.} \\
& +\frac{l_{a b} \sqrt{\pi}}{\lambda_{a b}}\left\{1-\frac{2\left(\frac{r_{a b}}{\lambda_{a b}}\right)}{\sqrt{\pi}}{ }_{1} F_{1}\left(\frac{1}{2}, \frac{3}{2},-\frac{r_{a b}^{2}}{\lambda_{a b}^{2}}\right)\right\}+\pi \lambda_{a b} l_{a b} \delta_{a b} \\
& \left.\times \exp \left(-\frac{r_{a b}^{2}}{\lambda_{a b}^{2}}\right)\left\{\left(\frac{4 \pi}{\lambda_{a b}^{2}}\right)^{\frac{3}{2}}+\frac{16 \pi}{9 \lambda_{a b}^{3}}{ }_{1} F_{1}\left(-1,3 / 2, \frac{r_{a b}^{2}}{\lambda_{a b}^{2}}\right)\right\}\right] \\
& \times \exp \left(\frac { l _ { a b } } { r } ( 1 + \operatorname { e x p } ( - \chi r _ { a b } ) ) \left[1+\sum_{d} n_{d}\right.\right. \\
& \times\left\{-\frac{\pi l_{a b}^{2}}{4 \chi} \cosh \left(\chi r_{a b}\right)+\frac{(-1)^{4 s}}{(2 s+1)^{2}} \frac{2 \sqrt{\pi} \exp \left(-\frac{r_{a b}^{2}}{\lambda_{a c}^{2}+\lambda_{b c}^{2}}\right)}{\left(\lambda_{a c}^{2}+\lambda_{b c}^{2}\right)^{\frac{3}{2}}} \lambda_{b c}^{3} \lambda_{a c}^{3} \delta_{a c} \delta_{b c}\right\} \\
& \left.\left.-\frac{\pi l_{a c} l_{b d} l_{c d}}{16 \chi^{3}}\left(\cosh \left(\chi r_{a b}\right)-\chi \sinh \left(\chi r_{a b}\right)\right)\right\}+\ldots\right] . \\
& \frac{1}{2} \sum_{c} \sum_{d} n_{c} n_{d}\left\{\frac{\pi^{2} l_{a c} l_{b c} l_{a d} l_{b d}}{16 \chi^{2}} \cosh { }^{2}(\chi r)\right.
\end{aligned}
$$

We can get another form of the quantum binary distribution function in the following form:

$$
F_{a b}^{q u}=S_{a b} \exp \left(\beta V_{a b}\right) F_{a b}^{c l},
$$

where

$$
\begin{aligned}
F_{a b}^{c l}= & 1+l_{a b} \frac{\exp (-\chi r)}{r}+l_{a b}^{2} \frac{\exp (-2 \chi r)}{2 r^{2}}+l_{a b}^{3} \frac{\exp (-3 \chi r)}{3 ! r^{3}}+l_{a b}^{4} \frac{\exp (-4 \chi r)}{4 ! r^{4}} \\
& -\pi\left(1+l_{a b} \frac{\exp (-\chi r)}{r}\right) \sum_{c} n_{c}\left(l_{a c} l_{b c}^{c}+l_{b c} l_{a c}^{2}\right)
\end{aligned}
$$




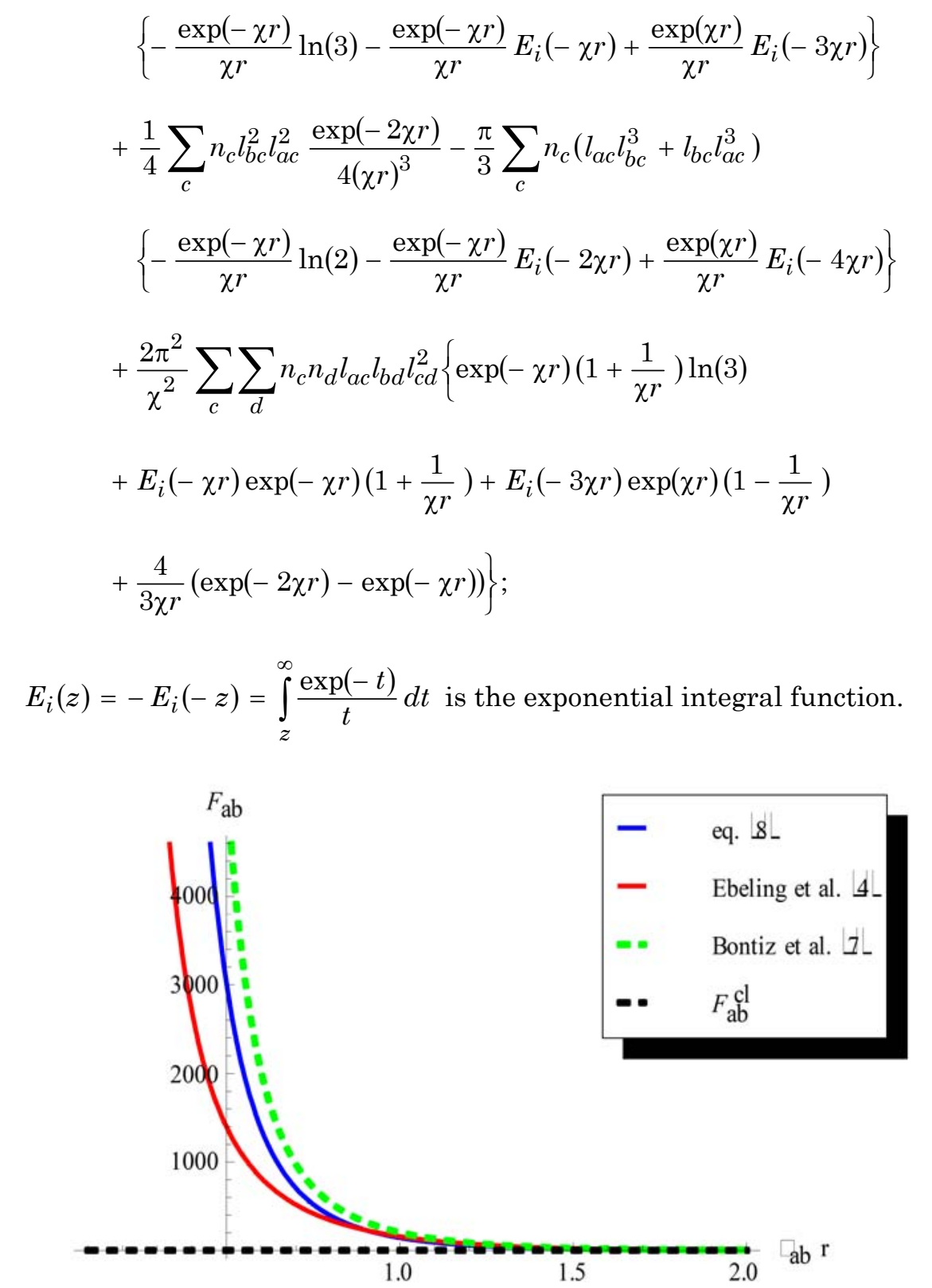

Figure 1. The comparison between the classical BDF, quantum BDF of Ebeling et al. [4], quantum BDF of Bontiz et al. [7] and our quantum BDF equation (8). 


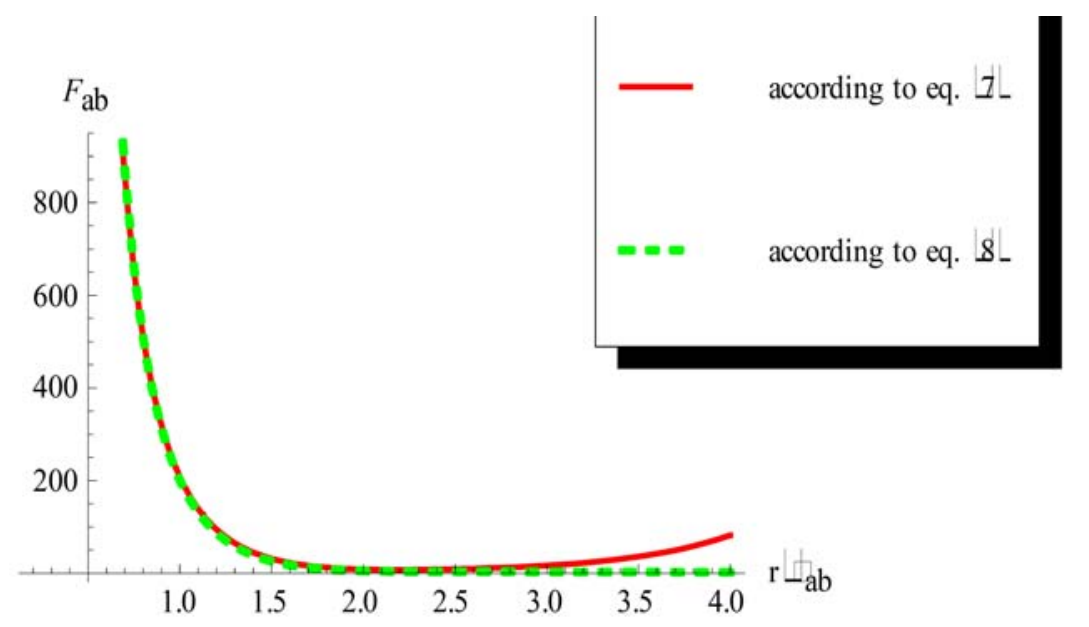

Figure 2. The comparison between the quantum BDF from Equation (7) and Equation (8).

\section{The Quantum Binary Distribution Function for Two Component Plasma}

The model under consideration is the two component plasma (TCP), i.e., neutral system of point like particles of positive and negative charges. For the numerical calculation, we restrict ourselves to the case of two component plasma which anti-symmetric with respect to the charges $e_{e}=-e_{i}=-e$ and therefore symmetrical with respect to the densities $n_{i}=n_{e}=n$. The binary distribution function for two component plasma is given by

$$
\begin{aligned}
F_{e e}^{q u}= & \exp \left(\frac { l _ { e e } } { r } ( 1 + \operatorname { e x p } ( - \chi r _ { e e } ) ) \left[1-\frac{1}{2} \exp \left(-\frac{r_{e e}^{2}}{\lambda_{e e}^{2}}\right)+\frac{l_{e e}}{r}\left\{1-\exp \left(-\frac{r_{e e}^{2}}{\lambda_{e e}^{2}}\right)\right\}\right.\right. \\
& +\frac{l_{e e} \sqrt{\pi}}{\lambda_{e e}}\left\{1-\frac{2\left(\frac{r_{e e}}{\lambda_{e e}}\right)}{\sqrt{\pi}}{ }_{1} F_{1}\left(\frac{1}{2}, \frac{3}{2},-\frac{r_{e e}^{2}}{\lambda_{e e}^{2}}\right)\right\} \\
& \left.+\pi \lambda_{e e} l_{e e} \exp \left(-\frac{r_{e e}^{2}}{\lambda_{e e}^{2}}\right)\left\{\left(\frac{4 \pi}{\lambda_{e e}^{2}}\right)^{\frac{3}{2}}+\frac{16 \pi \Gamma\left(\frac{5}{2}\right)}{9 \pi_{e e}^{3}} F_{1}\left(-1,3 / 2, \frac{r_{e e}^{2}}{\lambda_{e e}^{2}}\right)\right\}\right]
\end{aligned}
$$


THE QUANTUM BINARY AND TRIPLET DISTRIBUTION ...

9

$$
\begin{aligned}
& {\left[1+\sum_{e} n_{e}\left[-\frac{\pi l_{e e}^{2}}{4 \chi} \cosh \left(\chi r_{e e}\right)+\frac{(-1)^{4 s}}{(2 s+1)^{2}} \frac{2 \sqrt{\frac{\pi}{2}} \exp \left(-\frac{r_{e e}^{2}}{2 \lambda_{e e}^{2}}\right)}{\lambda_{e e}^{3}} \lambda_{e e}^{6}\right]\right.} \\
& +\frac{1}{2}\left(\sum_{e} n_{e}\right)^{2}\left[\frac{\pi^{2} l_{e e}^{4}}{16 \chi^{2}} \cosh ^{2}\left(\chi r_{e e}\right)-\frac{\pi l_{e e}^{3}}{16 \chi^{3}}\left\{\cosh \left(\chi r_{e e}\right)-\chi \sinh \left(\chi r_{e e}\right)\right\}\right] \\
& F_{i i}^{q u}=\exp \left(\frac { l _ { i i } } { r } ( 1 + \operatorname { e x p } ( - \chi r _ { i i } ) ) \left[1-\frac{1}{2} \exp \left(-\frac{r_{i i}^{2}}{\lambda_{i i}^{2}}\right)+\frac{l_{i i}}{r}\left\{1-\exp \left(-\frac{r_{i i}^{2}}{\lambda_{i i}^{2}}\right)\right\}\right.\right. \\
& +\frac{l_{i i} \sqrt{\pi}}{\lambda_{i i}}\left\{1-\frac{2\left(\frac{r_{i i}}{\lambda_{i i}}\right)}{\sqrt{\pi}}{ }_{1} F_{1}\left(\frac{1}{2}, \frac{3}{2},-\frac{r_{i i}^{2}}{\lambda_{i i}^{2}}\right)\right\} \\
& \left.+\pi \lambda_{i i} l_{i i} \exp \left(-\frac{r_{i i}^{2}}{\lambda_{i i}^{2}}\right)\left\{\left(\frac{4 \pi}{\lambda_{i i}^{2}}\right)^{\frac{3}{2}}+\frac{16 \pi \Gamma\left(\frac{5}{2}\right)}{9 \lambda_{i i}^{3}}{ }_{1} F_{1}\left(-1,3 / 2, \frac{r_{i i}^{2}}{\lambda_{i i}^{2}}\right)\right\}\right] \\
& {\left[1+\sum_{i} n_{i}\left\{-\frac{\pi l_{i i}^{2}}{4 \chi} \cosh \left(\chi r_{i i}\right)+\frac{(-1)^{4 s}}{(2 s+1)^{2}} \frac{2 \sqrt{\frac{\pi}{2}} \exp \left(-\frac{r_{i i}^{2}}{2 \lambda_{i i}^{2}}\right)}{\lambda_{i i}^{3}} \lambda_{i i}^{6}\right\}\right.} \\
& \left.+\frac{1}{2}\left(\sum_{e} n_{i}\right)^{2}\left[\frac{\pi^{2} l_{i i}^{4}}{16 \chi^{2}} \cosh ^{2}\left(\chi r_{i i}\right)-\frac{\pi l_{i i}^{3}}{16 \chi^{3}}\left\{\cosh \left(\chi r_{i i}\right)-\chi \sinh \left(\chi r_{i i}\right)\right\}\right]+\ldots\right] \\
& F_{e i}^{q u}=\left[1+\frac{l_{e i}}{r}\left\{1-\exp \left(-\frac{r_{e i}^{2}}{\lambda_{e i}^{2}}\right)\right\}+\frac{l_{e i} \sqrt{\pi}}{\lambda_{e i}}\left\{1-\frac{2\left(\frac{r_{e i}}{\lambda_{e i}}\right)}{\sqrt{\pi}}{ }_{1} F_{1}\left(\frac{1}{2}, \frac{3}{2},-\frac{r_{e i}^{2}}{\lambda_{e i}^{2}}\right)\right\}\right] \\
& \times \exp \left(\frac{l_{e i}}{r}\left(1+\exp \left(-\chi r_{e i}\right)\right)\right)\left[1-\frac{\pi l_{i e} l_{e e}}{4 \chi} \sum_{e} n_{e} \cosh \left(\chi r_{e i}\right)+\frac{1}{2} \sum_{i} \sum_{e} n_{i} n_{e}\right. \\
& \left.\times\left\{\frac{\pi^{2} l_{i e}^{2} l_{e e}^{2}}{16 \chi^{2}} \cosh ^{2}\left(\chi r_{e i}\right)-\frac{\pi l_{i e} l_{e e}^{2}}{16 \chi^{3}}\left(\cosh \left(\chi r_{e i}\right)-\sinh \left(\chi r_{e i}\right)\right)\right\}\right]
\end{aligned}
$$


where $F_{e e}^{q u}, F_{e i}^{q u}$, and $F_{i i}^{q u}$ are the quantum binary distribution functions for the indicated species.

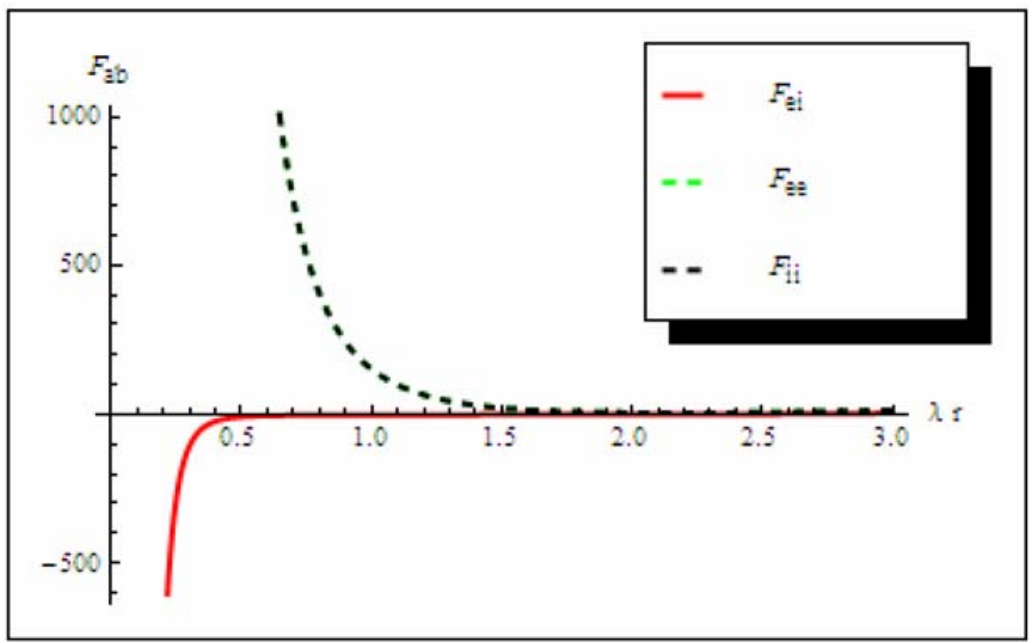

Figure 3. The comparison between $F_{e e}^{q u}, F_{e i}^{q u}$, and $F_{i i}^{q u}$ for TCP.

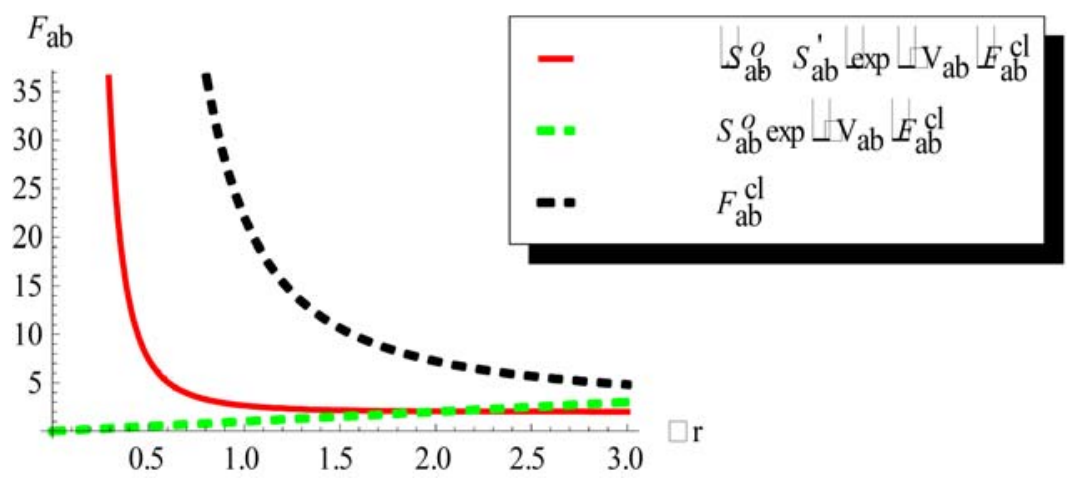

Figure 4. The comparison between the classical BDF and the quantum BDF for two component plasma.

\section{The Quantum Triplet Distribution Function (TDF)}

The quantum TDF is given by [9], which can be rewritten in the following form: 
THE QUANTUM BINARY AND TRIPLET DISTRIBUTION ...

$$
\begin{aligned}
F_{a b c}^{q u}= & S_{a b} S_{a c} S_{b c} \exp \left(g_{a b}+g_{a c}+g_{b c}+\beta\left(V_{a b}+V_{a c}+V_{b c}\right)\right) \\
& {\left[1+\sum_{d} n_{d} \int\left[f_{a d} f_{b d} f_{c d}+3 f_{a d} f_{b d}-3 g_{a d} g_{b d}\right] d r_{d}\right.} \\
& +\frac{1}{2} \sum_{d} \sum_{l} n_{d} n_{l} \iint d r_{d} d r_{l}\left[f_{a d} f_{b d} f_{c d} f_{a l} f_{b l} f_{c l} f_{d l}+6 f_{a d} f_{b d} f_{c d} f_{a l} f_{b l} f_{d l}\right. \\
& +18 f_{a l} f_{b d} f_{c l} f_{d l}+6 f_{c d} f_{c l} f_{a l} f_{b l} f_{d l}-6 f_{a d} f_{b d} f_{a l} f_{b l} f_{c l} f_{d l}-6 f_{a d} f_{b d} f_{b l} f_{c l} \\
& +9 f_{a d} f_{c d} f_{a l} f_{b l} f_{d l}-18 f_{a d} f_{a l} f_{b l}-9 f_{a d} f_{a l}-12 f_{c d} g_{a l} g_{d l}-18 f_{a d} f_{c l} g_{a l} g_{d l} \\
& -6 f_{a l} f_{b l} g_{a d} g_{c l} g_{d l}+6 f_{a l} f_{c d} f_{d l}+6 g_{b l} g_{c d} g_{d l}-18 g_{a d} g_{c d} f_{a l} f_{b l} \\
& \left.\left.-6 g_{c d} g_{a d} f_{a l} f_{b l} f_{c l}+9 g_{a d} g_{b d} g_{a l} g_{b l}\right]+\ldots\right] .
\end{aligned}
$$

The quantum triplet distribution function up to $O(n)$ is given by

$$
\begin{gathered}
F_{a b c}^{q u}\left(r, r^{\prime} r^{\prime \prime}\right)=S_{a b}(r) S_{a c}\left(r^{\prime}\right) S_{b c}\left(r^{\prime \prime}\right) \exp \left[g_{a b}(r)+g_{a c}\left(r^{\prime}\right)+g_{b c}\left(r^{\prime \prime}\right)+\beta\left\{V_{a b}(r)\right.\right. \\
\left.\left.+V_{a c}\left(r^{\prime}\right)+V_{b c}\left(r^{\prime \prime}\right)\right\}\right]\left[1+\sum_{d} n_{d}\left\{\frac{-3(-1)^{2 s_{a}}}{2 S_{a}+1} \sqrt{\pi^{3}} \lambda_{a b}^{3} \delta_{a b}-2 \pi l_{a b} \lambda_{a b}^{2}\right.\right. \\
+\frac{3}{2} \pi \sqrt{\pi} l_{a b}^{2} \lambda_{a b}-6 \pi l_{a b} \lambda_{a b}^{2} \delta_{a b} \frac{(-1)^{2 s_{a}}}{\left(2 S_{a}+1\right)}-3 \pi \sqrt{\pi}(\ln 2) l_{a b}^{2} \lambda_{a b} \delta_{a b} \\
\left.\left.\frac{(-1)^{2 s_{a}}}{2 S_{a}+1}-3 \frac{(-1)^{4 s}}{\left(2 s_{a}+1\right)^{2}} \frac{2 \sqrt{\pi} \exp \left(-\frac{r^{2}}{\lambda_{a c}^{2}+\lambda_{b c}^{2}}\right)}{\left(\lambda_{a c}^{2}+\lambda_{b c}^{2}\right)^{\frac{3}{2}}} \lambda_{b c}^{3} \lambda_{a c}^{3} \delta_{a c} \delta_{b c}\right\}+\ldots\right]
\end{gathered}
$$

Owing to Kirkwood superposition approximation (KSA), we can get the quantum triplet distribution function in the following form:

$$
F_{a b c}^{q u}=F_{a b}^{q u}(r) F_{b c}^{q u}\left(r^{\prime \prime}\right) F_{a c}^{q u}\left(r^{\prime}\right) .
$$

Expansion (15) is equivalent to assuming that the triplet distribution functions as a product of three radial distribution functions, substituting Equation (7) into expansion (15), we can get the triplet distribution function until $O\left(e^{8}\right)$ in the following form: 


$$
\begin{aligned}
& F_{a b c}^{q u}=\exp \left(\frac { l _ { a b } } { r _ { a b } } \left(1+\exp \left(-\chi r_{a b}\right)+\frac{l_{a c}}{r_{a c}}\left(1+\exp \left(-\chi r_{a c}\right)+\frac{l_{b c}}{r_{b c}}\left(1+\exp \left(-\chi r_{b c}\right)\right)\right.\right.\right. \\
& \times\left[1-\frac{1}{2} \delta_{a b} \exp \left(-\frac{r_{a b}^{2}}{\lambda_{a b}^{2}}\right)+\frac{l_{a b}}{r}\left\{1-\exp \left(-\frac{r_{a b}^{2}}{\lambda_{a b}^{2}}\right)\right\}\right. \\
& +\frac{l_{a b} \sqrt{\pi}}{\lambda_{a b}}\left\{1-\frac{2\left(\frac{r_{a b}}{\lambda_{a b}}\right)}{\sqrt{\pi}}{ }_{1} F_{1}\left(\frac{1}{2}, \frac{3}{2},-\frac{r_{a b}^{2}}{\lambda_{a b}^{2}}\right)\right\}+\pi \lambda_{a b} l_{a b} \delta_{a b} \exp \left(-\frac{r_{a b}^{2}}{\lambda_{a b}^{2}}\right) \\
& \left.\times\left\{\left(\frac{4 \pi}{\lambda_{a b}^{2}}\right)^{\frac{3}{2}}+\frac{16 \pi \Gamma\left(\frac{5}{2}\right)}{9 \lambda_{a b}^{3}} F_{1}\left(-1,3 / 2, \frac{r_{a b}^{2}}{\lambda_{a b}^{2}}\right)\right\}\right] \\
& \times\left[1-\frac{1}{2} \delta_{b c} \exp \left(-\frac{r_{b c}^{2}}{\lambda_{b c}^{2}}\right)+\frac{l_{b c}}{r_{b c}}\left\{1-\exp \left(-\frac{r_{b c}^{2}}{\lambda_{b c}^{2}}\right)\right\}\right. \\
& +\frac{l_{b c} \sqrt{\pi}}{\lambda_{b c}}\left\{1-\frac{2\left(\frac{r_{b c}}{\lambda_{b c}}\right)}{\sqrt{\pi}}{ }_{1} F_{1}\left(\frac{1}{2}, \frac{3}{2},-\frac{r_{b c}^{2}}{\lambda_{b c}^{2}}\right)\right\} \\
& \left.+\pi \lambda_{b c} l_{b c} \delta_{b c} \exp \left(-\frac{r_{b c}^{2}}{\lambda_{b c}^{2}}\right)\left\{\left(\frac{4 \pi}{\lambda_{b c}^{2}}\right)^{\frac{3}{2}}+\frac{16 \pi \Gamma\left(\frac{5}{2}\right)}{9 \lambda_{b c}^{3}}{ }_{1} F_{1}\left(-1,3 / 2, \frac{r_{b c}^{2}}{\lambda_{b c}^{2}}\right)\right\}\right] \\
& \times\left[1-\frac{1}{2} \delta_{a c} \exp \left(-\frac{r_{a c}^{2}}{\lambda_{a c}^{2}}\right)+\frac{l_{a c}}{r_{a c}}\left\{1-\exp \left(-\frac{r_{a c}^{2}}{\lambda_{a c}^{2}}\right)\right\}\right. \\
& +\frac{l_{a c} \sqrt{\pi}}{\lambda_{a c}}\left\{1-\frac{2\left(\frac{r}{\lambda_{a c}}\right)}{\sqrt{\pi}}{ }_{1} F_{1}\left(\frac{1}{2}, \frac{3}{2},-\frac{r_{a c}^{2}}{\lambda_{a c}^{2}}\right)\right\}+\pi \lambda_{a c} l_{a c} \delta_{a c} \exp \left(-\frac{r_{a c}^{2}}{\lambda_{a c}^{2}}\right) \\
& \left.\times\left\{\left(\frac{4 \pi}{\lambda_{a c}^{2}}\right)^{\frac{3}{2}}+\frac{16 \pi \Gamma\left(\frac{5}{2}\right)}{9 \lambda_{a c}^{3}}{ }_{1} F_{1}\left(-1,3 / 2, \frac{r_{a c}^{2}}{\lambda_{a c}^{2}}\right)\right\}\right] \\
& \times\left[1+\sum_{d} n_{d}\left\{-\frac{\pi l_{a b}^{2}}{4 \chi} \cosh \left(\chi r_{a b}\right)-\frac{\pi l_{a c}^{2}}{4 \chi} \cosh \left(\chi r_{a c}\right)-\frac{\pi l_{b c}^{2}}{4 \chi} \cosh \left(\chi r_{b c}\right)\right.\right.
\end{aligned}
$$




$$
\begin{gathered}
+\frac{(-1)^{4 s}}{(2 s+1)^{2}} \frac{2 \sqrt{\pi} \exp \left(-\frac{r_{a b}^{2}}{\lambda_{a c}^{2}+\lambda_{b c}^{2}}\right)}{\left(\lambda_{a c}^{2}+\lambda_{b c}^{2}\right)^{\frac{3}{2}}} \lambda_{b c}^{3} \lambda_{a c}^{3} \delta_{a c} \delta_{b c} \\
+\frac{(-1)^{4 s}}{(2 s+1)^{2}} \frac{2 \sqrt{\pi} \exp \left(-\frac{r_{a c}^{2}}{\lambda_{a b}^{2}+\lambda_{b c}^{2}}\right)}{\left(\lambda_{a b}^{2}+\lambda_{b c}^{2}\right)^{\frac{3}{2}}} \lambda_{b c}^{3} \lambda_{a b}^{3} \delta_{a b} \delta_{b c} \\
\left.+\frac{(-1)^{4 s}}{(2 s+1)^{2}} \frac{2 \sqrt{\pi} \exp \left(-\frac{r_{b c}^{2}}{\lambda_{a c}^{2}+\lambda_{a b}^{2}}\right)}{\left(\lambda_{a c}^{2}+\lambda_{b c}^{2}\right)^{\frac{3}{2}}} \lambda_{a b}^{3} \lambda_{a c}^{3} \delta_{a c} \delta_{a b}\right\}+\frac{9}{2} \sum_{d} \sum_{l} n_{d} n_{l} \\
\left.\times\left\{\frac{\pi^{2} l_{a l} l_{b l} l_{a d} l_{b d}}{16 \chi^{2}} \cosh ^{2}\left(\chi r_{a b}\right)-\frac{\pi l_{a l} l_{b d} l_{c d}}{16 \chi^{3}}\left(\cosh \left(\chi r_{a b}\right)-\chi \sinh \left(\chi r_{a b}\right)\right)\right\}+\ldots\right] .
\end{gathered}
$$

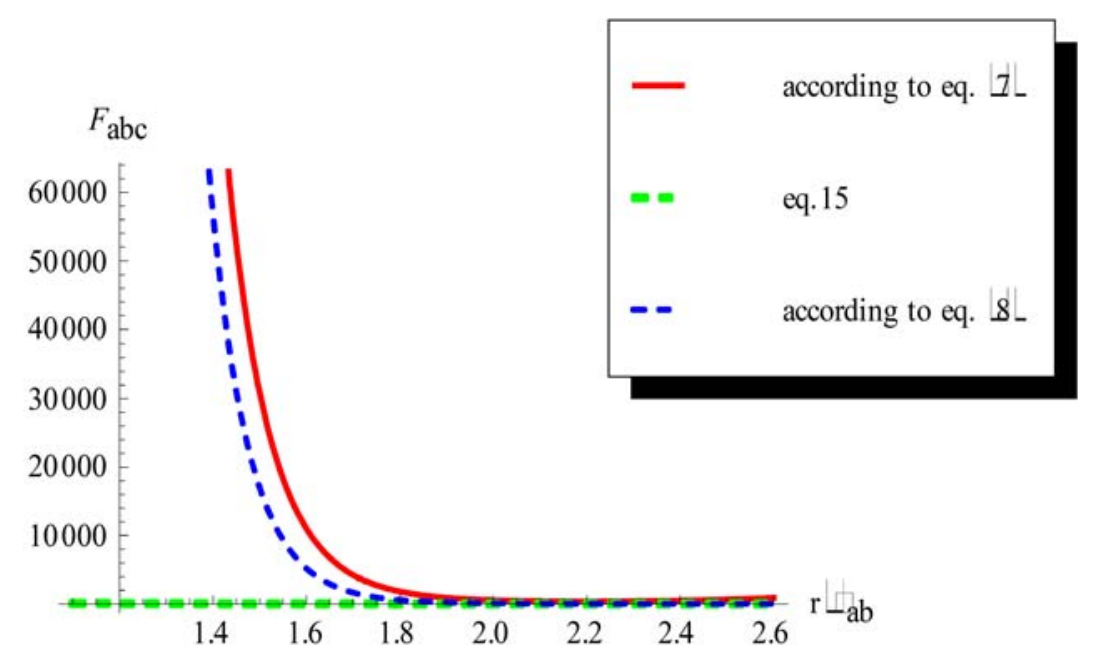

Figure 5. The comparison between TDF equation (15) and TDF owing to KSA (according to Equation (8) and Equation (7)). 


\section{The Quantum Triplet Distribution Function for Two Component Plasma}

From Equation (16), we can obtain the quantum TDF for two component plasma in the following form:

$$
\begin{aligned}
& F_{\text {eee }}^{q u}=\left(1-\frac{1}{2} \exp \left(-\frac{r_{e e}^{2}}{\lambda_{e e}^{2}}\right)+\frac{l_{e e}}{r}\left\{1-\exp \left(-\frac{r_{e e}^{2}}{\lambda_{e e}^{2}}\right)\right\}\right. \\
& +\frac{l_{e e} \sqrt{\pi}}{\lambda_{e e}}\left\{1-\frac{2\left(\frac{r_{e e}}{\lambda_{e e}}\right)}{\sqrt{\pi}}{ }_{1} F_{1}\left(\frac{1}{2}, \frac{3}{2},-\frac{r_{e e}^{2}}{\lambda_{e e}^{2}}\right)\right\} \\
& \left.+\pi \lambda_{e e} l_{e e} \exp \left(-\frac{r_{e e}^{2}}{\lambda_{e e}^{2}}\right)\left\{\left(\frac{4 \pi}{\lambda_{e e}^{2}}\right)^{\frac{3}{2}}+\frac{16 \pi \Gamma\left(\frac{5}{2}\right)}{9 \lambda_{e e}^{3}}{ }_{1} F_{1}\left(-1,3 / 2, \frac{r_{e e}^{2}}{\lambda_{e e}^{2}}\right)\right\}\right)^{3} \\
& \times \exp \left(\frac{3 l_{e e}}{r_{e e}}\left(1+\exp \left(-\chi r_{e e}\right)\right)\right. \\
& \times\left[1+\sum_{e} n_{e}\left\{-3 \frac{\pi l_{e e}^{2}}{4 \chi} \cosh \left(\chi r_{e e}\right)+\frac{3(-1)^{4 s}}{(2 s+1)^{2}} \sqrt{\frac{\pi}{2}} \exp \left(-\frac{r_{e e}^{2}}{2 \lambda_{e e}^{2}}\right) \lambda_{e e}^{3}\right\}\right. \\
& \left.+\frac{1}{2}\left(\sum_{e} n_{e}\right)^{2}\left\{\frac{\pi^{2} l_{e e}^{4}}{16 \chi^{2}} \cosh ^{2}\left(\chi r_{e e}\right)-\frac{\pi l_{e e}^{3}}{16 \chi^{3}}\left(\cosh \left(\chi r_{e e}\right)-\chi \sinh \left(\chi r_{e e}\right)\right)\right\}+\ldots\right] \\
& F_{i i i}^{q u}=\left(1-\frac{1}{2} \exp \left(-\frac{r_{i i}^{2}}{\lambda_{i i}^{2}}\right)+\frac{l_{i i}}{r}\left\{1-\exp \left(-\frac{r_{i i}^{2}}{\lambda_{i i}^{2}}\right)\right\}\right. \\
& +\frac{l_{i i} \sqrt{\pi}}{\lambda_{i i}}\left\{1-\frac{2\left(\frac{r_{i i}}{\lambda_{i i}}\right)}{\sqrt{\pi}}{ }_{1} F_{1}\left(\frac{1}{2}, \frac{3}{2},-\frac{r_{i i}^{2}}{\lambda_{i i}^{2}}\right)\right\} \\
& \left.+\pi \lambda_{i i} l_{i i} \exp \left(-\frac{r_{i i}^{2}}{\lambda_{i i}^{2}}\right)\left\{\left(\frac{4 \pi}{\lambda_{i i}^{2}}\right)^{\frac{3}{2}}+\frac{16 \pi \Gamma\left(\frac{5}{2}\right)}{9 \lambda_{i i}^{3}}{ }_{1 i} F_{1}\left(-1,3 / 2, \frac{r_{i i}^{2}}{\lambda_{i i}^{2}}\right)\right\}\right)^{3}
\end{aligned}
$$




$$
\begin{aligned}
& \times \exp \left(\frac{3 l_{i i}}{r_{i i}}\left(1+\exp \left(-\chi r_{i i}\right)\right)\right. \\
& \times\left[1+\sum_{i} n_{i}\left\{-3 \frac{\pi l_{i i}^{2}}{4 \chi} \cosh \left(\chi r_{i i}\right)+\frac{3(-1)^{4 s}}{(2 s+1)^{2}} \sqrt{\frac{\pi}{2}} \exp \left(-\frac{r_{i i}^{2}}{2 \lambda_{i i}^{2}}\right) \lambda_{i i}^{3}\right\}\right. \\
& \left.+\frac{1}{2}\left(\sum_{i} n_{i}\right)^{2}\left\{\frac{\pi^{2} l_{i i}^{4}}{16 \chi^{2}} \cosh ^{2}\left(\chi r_{i i}\right)-\frac{\pi l_{i i}^{3}}{16 \chi^{3}}\left(\cosh \left(\chi r_{i i}\right)-\chi \sinh \left(\chi r_{i i}\right)\right)\right\}+\ldots\right] \text {; } \\
& F_{e e i}^{q u}=\left[1-\frac{1}{2} \exp \left(-\frac{r_{e e}^{2}}{\lambda_{e e}^{2}}\right)+\frac{l_{e e}}{r}\left\{1-\exp \left(-\frac{r_{e e}^{2}}{\lambda_{e e}^{2}}\right)\right\}+\frac{l_{e e} \sqrt{\pi}}{\lambda_{e e}}\left\{1-\frac{2\left(\frac{r_{e e}}{\lambda_{e e}}\right)}{\sqrt{\pi}}{ }_{1} F_{1}\left(\frac{1}{2}, \frac{3}{2},-\frac{r_{e e}^{2}}{\lambda_{e e}^{2}}\right)\right\}\right. \\
& \left.+\pi \lambda_{e e} l_{e e} \exp \left(-\frac{r_{e e}^{2}}{\lambda_{e e}^{2}}\right)\left\{\left(\frac{4 \pi}{\lambda_{e i}^{2}}\right)^{\frac{3}{2}}+\frac{16 \pi \Gamma\left(\frac{5}{2}\right)}{9 \lambda_{e i}^{3}}{ }_{e i} F_{1}\left(-1,3 / 2, \frac{r_{e e}^{2}}{\lambda_{e e}^{2}}\right)\right\}\right] \\
& \times\left(1+\frac{l_{e i}}{r_{e i}}\left\{1-\exp \left(-\frac{r_{e i}^{2}}{\lambda_{e i}^{2}}\right)\right\}+\frac{l_{e i} \sqrt{\pi}}{\lambda_{e i}}\left\{1-\frac{2\left(\frac{r}{\lambda_{e i}}\right)}{\sqrt{\pi}}{ }_{1} F_{1}\left(\frac{1}{2}, \frac{3}{2},-\frac{r_{e i}^{2}}{\lambda_{e i}^{2}}\right)\right\}\right)^{2} \\
& \times \exp \left(\frac { l _ { e e } } { r _ { e e } } \left(1+\exp \left(-\chi r_{e e}\right)+2 \frac{l_{e i}}{r}\left(1+\exp \left(-\chi r_{e i}\right)\right)\right.\right. \\
& \times\left[1+\sum_{d} n_{d}\left\{-\frac{\pi l_{e e}^{2}}{4 \chi} \cosh \left(\chi r_{e e}\right)-\frac{\pi l_{e i}^{2}}{4 \chi} \cosh \left(\chi r_{e i}\right)-\frac{\pi l_{e i}^{2}}{4 \chi} \cosh \left(\chi r_{e i}\right)\right\}\right. \\
& \left.+\frac{1}{2} \sum_{e} \sum_{e} n_{e} n_{e}\left\{\frac{\pi^{2} l_{e e}^{2}}{16 \chi^{2}} \cosh ^{2}\left(\chi r_{e e}\right)-\frac{\pi l_{a l} l_{b d} l_{c d}}{16 \chi^{3}}\left(\cosh \left(\chi r_{e e}\right)-\chi \sinh \left(\chi r_{e e}\right)\right)\right\}\right] \text {; } \\
& F_{i i e}^{q u}=\left[1-\frac{1}{2} \exp \left(-\frac{r_{i i}^{2}}{\lambda_{i i}^{2}}\right)+\frac{l_{i i}}{r}\left\{1-\exp \left(-\frac{r_{i i}^{2}}{\lambda_{i i}^{2}}\right)\right\}+\frac{l_{i i} \sqrt{\pi}}{\lambda_{i i}}\left\{1-\frac{2\left(\frac{r_{i i}}{\lambda_{i i}}\right)}{\sqrt{\pi}}{ }_{1} F_{1}\left(\frac{1}{2}, \frac{3}{2},-\frac{r_{i i}^{2}}{\lambda_{i i}^{2}}\right)\right\}\right. \\
& \left.+\pi \lambda_{i i} l_{i i} \exp \left(-\frac{r_{i i}^{2}}{\lambda_{i i}^{2}}\right)\left\{\left(\frac{4 \pi}{\lambda_{i i}^{2}}\right)^{\frac{3}{2}}+\frac{16 \pi \Gamma\left(\frac{5}{2}\right)}{9 \lambda_{i i}^{3}}{ }_{i i} F_{1}\left(-1,3 / 2, \frac{r_{i i}^{2}}{\lambda_{i i}^{2}}\right)\right\}\right]
\end{aligned}
$$




$$
\begin{aligned}
& \times\left[1+\frac{l_{i e}}{r_{i e}}\left\{1-\exp \left(-\frac{r_{i e}^{2}}{\lambda_{i e}^{2}}\right)\right\}+\frac{l_{i e} \sqrt{\pi}}{\lambda_{i e}}\left\{1-\frac{2\left(\frac{r_{i e}}{\lambda_{i e}}\right)}{\sqrt{\pi}}{ }_{1} F_{1}\left(\frac{1}{2}, \frac{3}{2},-\frac{r_{i e}^{2}}{\lambda_{i e}^{2}}\right)\right]^{2}\right. \\
& \times \exp \left(\frac { l _ { i i } } { r } \left(1+\exp \left(-\chi r_{i i}\right)+2 \frac{l_{i e}}{r}\left(1+\exp \left(-\chi r_{i e}\right)\right)\right.\right. \\
& \times\left[1+\sum_{e} n_{e}\left\{-\frac{\pi l_{i i}^{2}}{4 \chi} \cosh \left(\chi r_{i i}\right)-\frac{\pi l_{i e}^{2}}{2 \chi} \cosh \left(\chi r_{i e}\right)\right\}\right. \\
& +\frac{1}{2}\left(\sum_{e} n_{e}\right)^{2}\left\{\frac{\pi^{2} l_{i e}^{4}}{16 \chi^{2}} \cosh ^{2}\left(\chi r_{i i}\right)-\frac{\pi l_{i e}^{2} l_{e e}}{16 \chi^{3}}\left(\cosh \left(\chi r_{i i}\right)-\chi \sinh \left(\chi r_{i i}\right)\right)\right\},
\end{aligned}
$$

where $F_{e e e}^{q u}, F_{i i i}^{q u}, F_{e e i}^{q u}$, and $F_{i i e}^{q u}$ are the quantum triplet distribution functions for the indicated species.

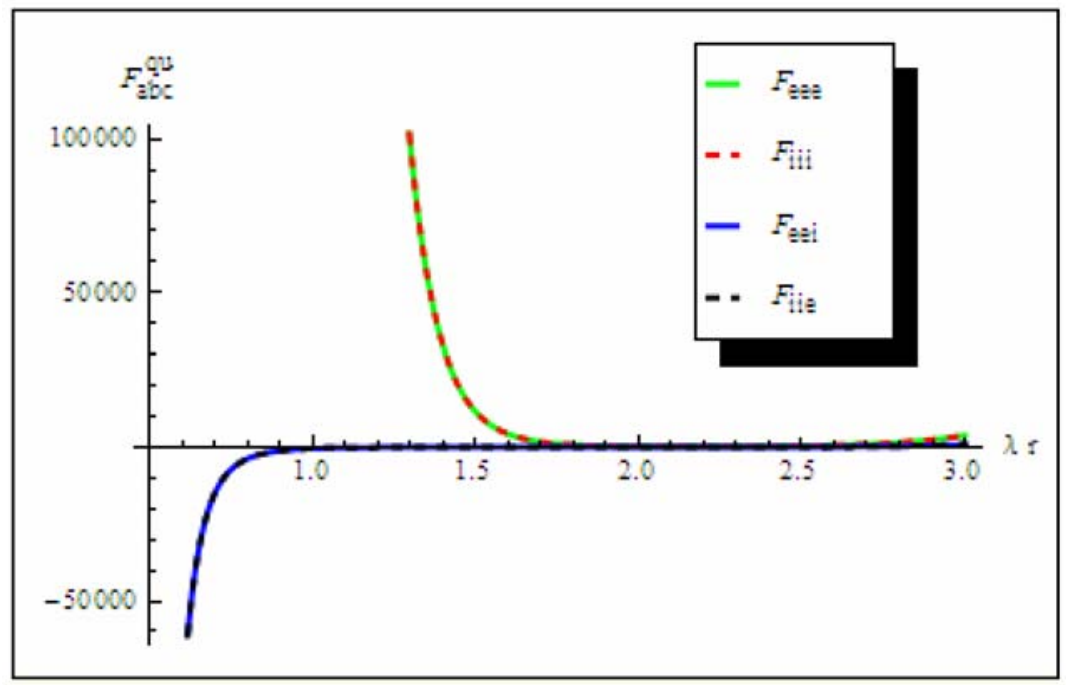

Figure 6. The comparison between $F_{e e e}^{q u}, F_{i i i}^{q u}, F_{e i i}^{q u}$, and $F_{i i e}^{q u}$ for TCP. 


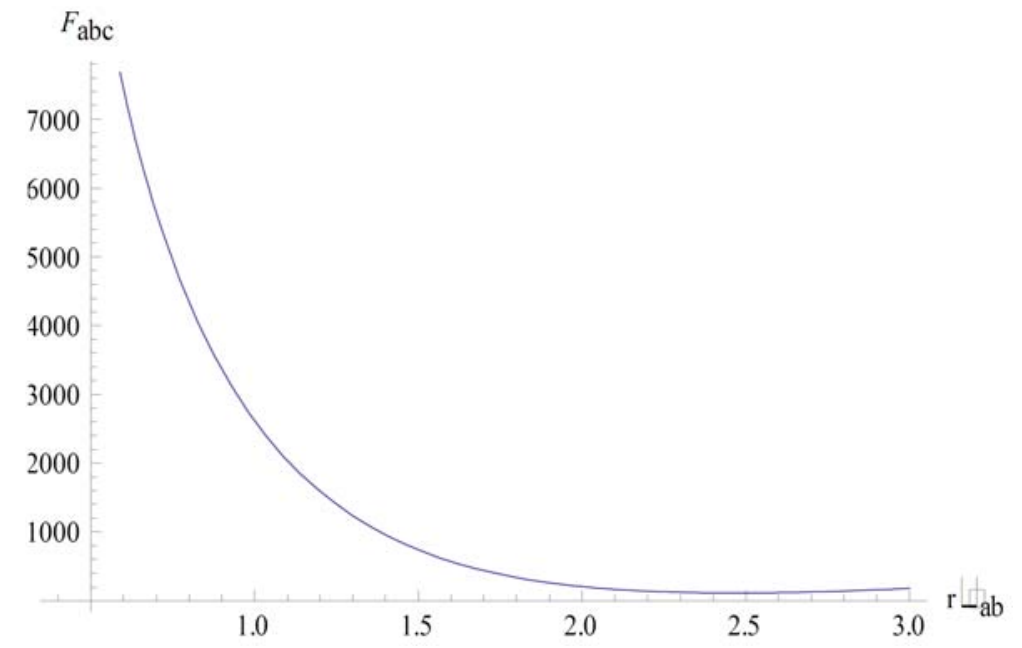

For one component plasma

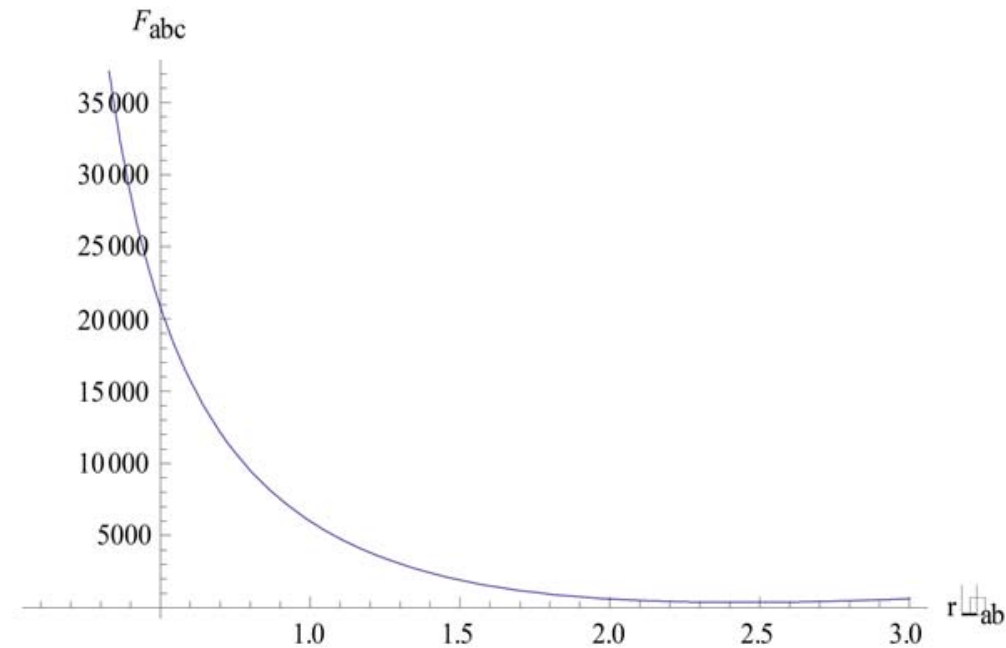

For two component plasma

Figure 7. The triplet distribution function (Equation (16)) at $r=r^{\prime}=r^{\prime \prime}$. 


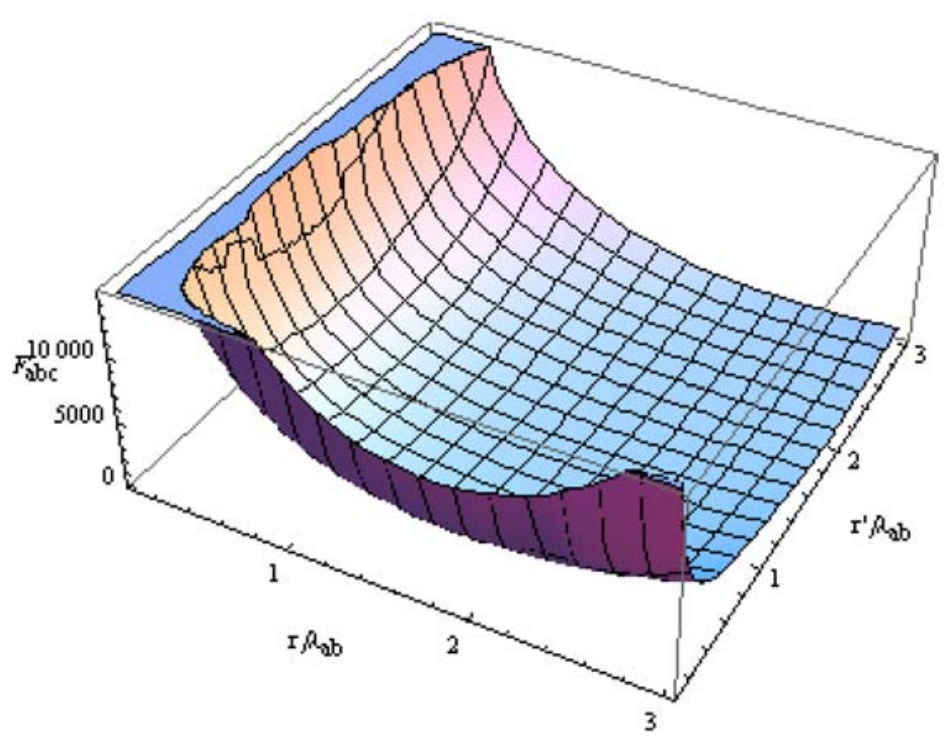

For one component plasma

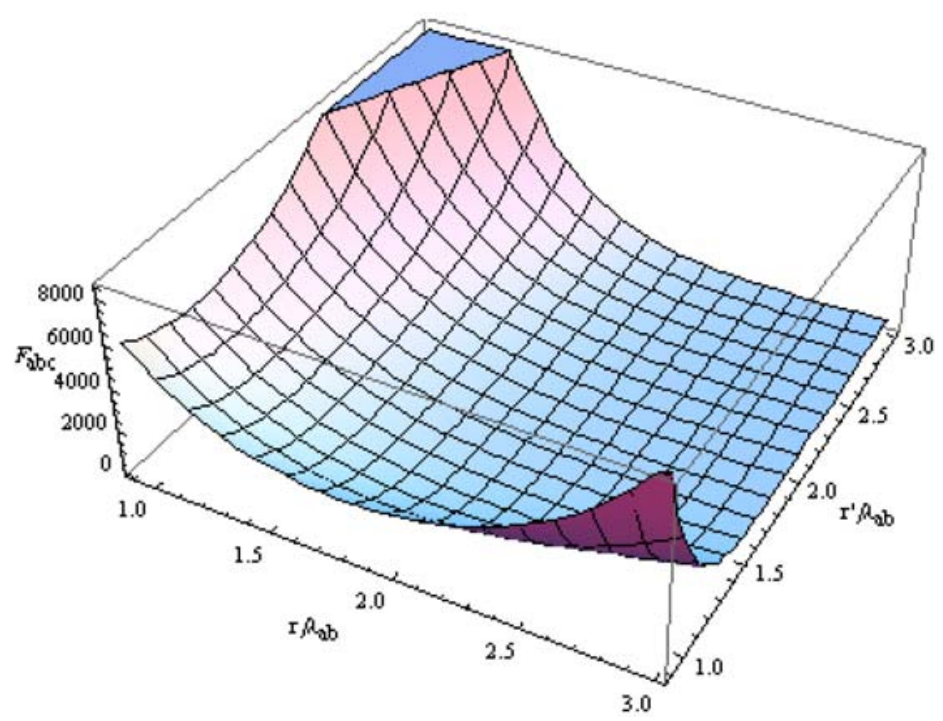

For two component plasma

Figure 8. The triplet distribution function (Equation (16)) at $r=r^{\prime} \neq r^{\prime \prime}$. 


\section{Conclusion}

In the previous paper [9], we investigated the density expansions of the reduced distribution functions of a multi-component plasma of charged particles that interact through the effective potential and obtained the binary and triplet distribution functions from the BBGKY hierarchy in the classical form. In this paper, we used the previous results to calculate the analytical forms of the quantum binary and triplet distribution functions for two component plasma; we obtained two forms of the quantum triplet distribution function one of them is based on the Kirkwood superposition approximation (KSA); which is consisting of the assumption that the potential in a set of three particles is the sum of the three pair potentials, this is equivalent to assuming that the triplet distribution function is the product of the three radial distribution functions, and the other form is calculated by integration of the triplet distribution function.

We plot the triplet distribution function for equilateral configurations $F_{a b c}(r, r, r)$ and for certain isosceles configurations $F_{a b c}\left(r, r, r^{\prime}\right)$ for a number of values of $r, r^{\prime}$, which are chosen to make possible a comparison. The triplet distribution function in $3 \mathrm{D}$ for one and two component plasma is given in Figure 8. The model under consideration is the two component plasma (TCP), i.e., neutral system of point like particles of positive and negative charges. For the numerical calculation, we restrict ourselves to the case of a two component plasma which is anti-symmetric with respect to the charges $\left(e_{e}=-e_{i}=-e\right)$ and symmetrical with respect to densities $n_{i}=n_{e}=n$.

\section{Acknowledgements}

The author wish to thank Professor Nagat Hussein for intensive discussions and advice. 


\section{References}

[1] J. G. Kirkwood, J. Chem. Phys. 2 (1935), 300.

[2] P. Ludwig, H. Thomsen, K. Balzer, A. Filinov and M. Bonitz, Plasma Phys. Control Fusion 52 (2010), 124013.

[3] H. B. Nersisyan and G. Zwicknagel, J. Phys. A 39 (2006), 4677.

[4] W. Ebeling and S. Sadykova, Contrib. Plasma Phys. 47 (2007), 659.

[5] K. Zahn, G. Maret, C. Rub and H. Grünberg, Phys. Rev. Lett. 91 (2003), 115502.

[6] C. Russ, M. Brunner, C. Bechinger and H. Grünberg, Europhys. Lett. 69 (2005), 468.

[7] M. Bonitz, C. Henning and D. Block, Reports on Progress in Physics 73 (2010), 066501.

[8] W. D. Kraeft, D. Kremp, W. Ebeling and G. Röpke, Quantum Statistics of Charged Particle Systems (Akademie Verlag, Berlin, 1986); W. Ebeling, G. Kelbg, H. Hoffmann, Beitr. Plasma Physik. 7 (1967), 233.

[9] D. A. Eisa, Contrib. Plasma Phys. 52 (2012), 261.

\section{Appendix A}

$$
\begin{aligned}
\int f_{a d} f_{b d} f_{c d} d r_{d}= & \frac{1}{(2 \pi)^{9 / 2}} \iiint \int d t_{1} d t_{2} d t_{3} d r_{d} \exp \left[i\left(t_{1}+t_{2}+t_{3}\right) \cdot r_{d}\right] \\
& \exp \left(i\left(t_{1} \cdot r_{a}+t_{2} \cdot r_{b}+t_{3} \cdot r_{c}\right)\right) \widetilde{F}\left(t_{1}\right) \widetilde{F}\left(t_{2}\right) \widetilde{F}\left(t_{3}\right) \\
= & (2 \pi)^{-9 / 2} \iiint d t_{1} d t_{2} d t_{3} \delta\left(t_{1}+t_{2}+t_{3}\right) \\
& \exp \left(i\left(t_{1} \cdot r_{a}+t_{2} \cdot r_{b}+t_{3} \cdot r_{c}\right)\right) \widetilde{F}\left(t_{1}\right) \widetilde{F}\left(t_{2}\right) \widetilde{F}\left(t_{3}\right) \\
= & (2 \pi)^{-9 / 2} \iint d t_{1} d t_{2} \exp \left(i\left(t_{1} \cdot r_{a c}+t_{2} \cdot r_{b c}\right)\right) \widetilde{F}\left(t_{1}\right) \tilde{F}\left(t_{2}\right) \widetilde{F}\left(-t_{1}-t_{2}\right) \\
= & \frac{16 \pi^{2}}{(2 \pi)^{9} / 2} \int_{0}^{\infty} \int_{0}^{\infty} \frac{t_{1} t_{2} \sin \left(t_{1} r_{a c}\right) \sin \left(t_{2} r_{b c}\right)}{r_{a c} r_{b c}} \widetilde{F}\left(t_{1}\right) \widetilde{F}\left(t_{2}\right) \widetilde{F}\left(-t_{1}-t_{2}\right) d t_{1} d t_{2},
\end{aligned}
$$




$$
\begin{aligned}
& \int d r_{c} d r_{d} f_{a c} f_{b d} f_{c d}=\frac{1}{(2 \pi)^{9 / 2}} \iiint d t_{1} d t_{2} d t_{3} d r_{c} d r_{d} \widetilde{F}\left(t_{1}\right) \widetilde{F}\left(t_{2}\right) \widetilde{F}\left(t_{3}\right) \\
& \exp \left(i t_{1} \cdot r_{a c}+i t_{2} \cdot r_{b d}+i t_{3} \cdot r_{c d}\right) \\
& =\frac{1}{(2 \pi)^{9 / 2}} \iiint d t_{1} d t_{2} d t_{3} d r_{c} d r_{d} \widetilde{F}\left(t_{1}\right) \widetilde{F}\left(t_{2}\right) \widetilde{F}\left(t_{3}\right) \\
& \exp \left(-i\left(t_{1} \cdot r_{a}+t_{2} \cdot r_{b}\right)\right) \\
& \exp \left(i\left(t_{3}+t_{2}\right) \cdot r_{d}\right) \exp \left(i\left(t_{3}+t_{1}\right) \cdot r_{c}\right) \\
& =\frac{1}{(2 \pi)^{9 / 2}} \iiint d t_{1} d t_{2} d t_{3} \tilde{F}\left(t_{1}\right) \tilde{F}\left(t_{2}\right) \tilde{F}\left(t_{3}\right) \\
& \exp \left(-i t_{1} \cdot r_{a}-i t_{2} \cdot r_{b}\right) \delta\left(t_{2}+t_{3}\right) \delta\left(-t_{1}+t_{3}\right) \\
& =\frac{1}{(2 \pi)^{9 / 2}} \iint d t_{1} d t_{3} \delta\left(-t_{1}+t_{3}\right) \tilde{F}\left(t_{1}\right) \tilde{F}\left(-t_{3}\right) \tilde{F}\left(t_{3}\right) \\
& \exp \left(-i\left(t_{1} \cdot r_{a}-t_{3} \cdot r_{b}\right)\right) \\
& =\frac{1}{(2 \pi)^{9 / 2}} \int d t_{1} \widetilde{F}\left(t_{1}\right) \tilde{F}\left(-t_{1}\right) \tilde{F}\left(t_{1}\right) \exp \left(i t_{1} . r_{a b}\right) \\
& =\frac{4 \pi}{(2 \pi) \frac{9}{2}} \int \frac{t \sin \left(t r_{a b}\right)}{r_{a b}} \widetilde{F}(t) \widetilde{F}(-t) \widetilde{F}(t) d t .
\end{aligned}
$$

